\title{
Selective Disruption of "Late Onset" Sagittal Banding Patterns by Ectopic Expression of Engrailed-2 in Cerebellar Purkinje Cells
}

\author{
Stephan L. Baader, ${ }^{1}$ Michael W. Vogel, ${ }^{2}$ Salih Sanlioglu, ${ }^{1}$ Xulun Zhang, ${ }^{1}$ and John Oberdick ${ }^{1}$ \\ ${ }^{1}$ Division of Neuroscience and the Neurobiotechnology Center, The Ohio State University College of Medicine, Columbus, \\ Ohio 43210, and 2 Maryland Psychiatric Research Center, University of Maryland Medical School, Baltimore, Maryland \\ 21228
}

To explore the role of Engrailed proteins in development of the cerebellum, Engrailed-2 (En-2) was ectopically expressed in cerebellar Purkinje cells from the late embryonic stage into adulthood. The fundamental organization of Purkinje cell sagittal zones as revealed by the "early onset" markers L7- $\beta$-gal and cadherin- 8 was found to be virtually identical to that in wild type. In contrast, "late onset" sagittal banding patterns revealed by Purkinje cell markers zebrin I, zebrin II, and 9-Oacetyl $\mathrm{G}_{\mathrm{D} 3}$ Ganglioside (P-Path), and the granule cell marker NADPH-diaphorase, were disrupted. In general, although some evidence of banding was still detectable, boundaries defined by the latter markers were poorly defined, and the patterns overall took on a diffuse appearance. In parallel with the changes in late onset markers, anterograde tracing of spinocerebellar axons revealed a general diffusion of the mossy fiber projection pattern in lobule VIII and the anterior lobe. These observations suggest that at least two separate mediolateral boundary systems exist in the cerebellum, and these are differentially affected by ectopic En-2 expression. Alternatively, one boundary system exists that remains primarily intact in the mutant, but recognition of this system by a set of late developmental events is perturbed.

Key words: mouse; cerebellum; Purkinje cell; Engrailed-2; mossy fibers; pcp-2(L7); cadherin; zebrin; NOS
The cerebellum is an ideal model system for studying the molecular and cellular mechanisms of boundary formation in the brain. It is divided into sagittally oriented compartments that can be visualized by patterns of afferent and efferent projection (Voogd and Bigaré, 1980), as well as by patterns of expression of a host of molecular markers (Hawkes et al., 1985; Brochu et al., 1990; Oberdick et al., 1993). The molecular record is complex because there are clearly differences in the pattern and timing of mediolateral boundaries in the cerebellum. For example, markers such as L7, calbindin, cadherin, and others ("early onset") show banded expression patterns beginning as early as embryonic day 15 (E15) (Wassef et al., 1985; Oberdick et al., 1993; Arndt et al., 1998). In contrast, markers exemplified by zebrin I and II ("late onset") are uniformly expressed during the early postnatal period, but this gives way to a pattern of stripes as the cerebellum matures (Leclerc et al., 1988).

The two major afferents to the cerebellum, climbing and mossy fibers, show a high degree of sagittal organization (Voogd and Bigaré, 1980; Arsénio-Nunes and Sotelo, 1985); the former synapse with Purkinje cells (the sole cortical output) and the latter with granule cells (major cortical interneurons). Like the molec-

\footnotetext{
Received Dec. 10, 1998; revised April 15, 1999; accepted April 22, 1999.

This work was supported by National Institutes of Health (NIH) Grant RO1NS33114 and National Science Foundation Grant IBN-9309611 to J.O., NIH Grant RO1-NS34309 to M.W.V., and Deutsche Forschungsgemeinschaft Research Stipend 1483/2-1 to S.L.B. Additional support was provided by the W. M. Keck Foundation to the Genetics Research Facility at The Ohio State University. We thank Alex Joyner for the En $2^{\text {hd }}$ null mutant mice, Richard Hawkes for providing the antizebrin I and II antibodies, and Mark Seeger and Christine Beattie for critical reading of this manuscript.

Drs. Baader and Vogel contributed equally to this work.

Correspondence should be addressed to Dr. John Oberdick, 190 Rightmire Hall, 1060 Carmack Road, The Ohio State University, Columbus, OH 43210.

Copyright (C) 1999 Society for Neuroscience $0270-6474 / 99 / 195370-10 \$ 05.00 / 0$
}

ular banding patterns, the acquisition of sagittal pattern within the mossy and climbing fiber systems also differs with respect to timing. Climbing fibers are clearly restricted to discrete sagittal territories within the cerebellar cortex during the late embryonic period in rodents (Paradies et al., 1996), whereas mossy fibers acquire this property gradually during the first postnatal week (Arsénio-Nunes and Sotelo, 1985).

Both early onset and late onset sagittal markers show boundaries that are primarily coincident with those of mossy and climbing fiber projections (Gravel et al., 1987; Gravel and Hawkes, 1990; Paradies et al., 1996). Examination of patterns of both early and late onset markers in organ culture and by transplantation, respectively, suggests a significant intrinsic component to the compartmental organization of the cerebellum (Wassef et al., 1990; Oberdick et al., 1993; Seil et al., 1995; Chédotal et al., 1996). Thus, despite the obvious differences in their development, there is no evidence of any mechanistic distinction between late onset versus early onset molecular banding patterns.

It has been suggested that the mammalian homologs of Drosophila segment polarity genes might be involved in organizing cerebellar compartments (Millen et al., 1995; for review, see Oberdick et al., 1998). This has been difficult to test because mutations in these genes result either in a deletion of most of the cerebellum or in very little effect, the latter presumably because of genetic compensation (Millen et al., 1994; Wurst et al., 1994). To extend such analyses, the Engrailed-2 protein (En-2) was ectopically expressed in the cerebellum of transgenic (L7En-2) mice using the Purkinje cell-specific pcp-2(L7) promoter. Using this gain-of-function approach, we find that the L7-type (early onset) and zebrin-type (late onset) markers behave differently when confronted with the same genetic perturbation, suggesting that at least two distinct boundary systems exist in the cerebellum. 


\section{MATERIALS AND METHODS}

Mouse strains and transgene constructions. Construction of the L7En-2 (line 20) (Baader et al., 1998) and L7- $\beta$-gal (line L7 $\beta$ G3) (Oberdick et al., 1993) transgenic mice have been described previously. These lines were maintained in $\mathrm{FVB} / \mathrm{N}$ and $\mathrm{B} 6 \mathrm{C} 3 \mathrm{~F} 1$ hybrid backgrounds, respectively. The effects on zebrin II banding described herein have been tested in both genetic strain backgrounds. The $E n-2^{\text {hd }}$ mutant mice were maintained on a $129 / \mathrm{Sv}$ inbred background as described previously (Vogel et al., 1996).

The L7 $\beta$ G3 line was originally reported to show uniform expression in cerebellum after postnatal day 10 (P10) (Oberdick et al., 1993). Although the embryonic staining pattern is identical to that observed previously, this line currently shows a striking banding pattern well into adult life. The reasons for this change are unknown.

Histochemistry and immunohistochemistry. The protocol used for immunohistochemistry of floating sections has been described previously (Baader et al., 1998). The blocking solution was 10\% goat serum in PBS, and the same solution was used to dilute the zebrin I or II antibodies (1:20; kindly provided by Dr. R. Hawkes, University of Calgary, Calgary, Alberta, Canada). Monoclonal anti-9- $O$-acetyl $\mathrm{G}_{\mathrm{D} 3}$ Ganglioside (P-Path) was from Sigma (cat \#G-8406). The binding of antibody was revealed by the Vectastain ABC kit as per manufacturer's instructions (Vector Laboratories, Burlingame, CA). For histochemical staining of sections for NADPH-diaphorase, the protocol described by Schilling et al. (1994) was used.

All sections were mounted in Permount and visualized using a Zeiss (Oberkochen, Germany) Axiophot microscope connected to standard computer equipment. Captured images were transferred to gray scale images, and dimensions were measured using IP Lab Spectrum (version 2.5.7; Signal Analytics Corporation, Vienna, VA).

For reconstruction of zebrin and P-Path banding patterns, every other section of wild-type and L7En-2 cerebellum was captured as a digitized image and transferred to Photoshop 4.0 (Adobe Systems, San Jose, CA). Using the polygon lasso tool, dorsal and ventral aspects of lobule VIII were assembled and aligned as shown in Figure 7 (in this figure, above the line is dorsal, and below is ventral).

In situ hybridization. Riboprobe synthesis, preparation of sections, and hybridization were performed as reported previously (Bian et al., 1996; Baader et al., 1998). The clone for visualizing cadherin-8 (Cad-8) expression was kindly provided by Dr. C. Redies (University Hospital Essen, Essen, Germany) and is described by Korematsu and Redies (1997). For generating the $\left[{ }^{35} \mathrm{~S}\right]$-labeled probe, this clone was linearized with HindIII, and antisense riboprobe was synthesized with T3 polymerase.

Spinal cord injections of wheat germ-agglutinin. Surgeries, injections, and tissue processing were as described previously using a modified TMB protocol to reveal HRP (DeOlmos et al., 1978; Vogel et al., 1996). For the L7En-2 mouse model, 9 wild-type and 12 transgenic mice (aged P25) from two litters were injected and processed for this analysis. Numbers of animals processed for $E n-2^{\text {hd }} / E n-2^{\text {hd }}$ null mutants was as described previously (Vogel et al., 1996).

TMB stained cerebellar sections were viewed with polarized light, and the images were digitized with a TARGA 2000 video board (True Vision, Santa Clara, CA) in a Power Macintosh 7300 . Every other section was digitized. The digitized images were imported into NIH Image and calibrated with a stage micrometer. The distribution of spinocerebellar mossy fiber terminals was reconstructed by first defining the midline of the cerebellum based on lobule shape and the HRP labeling pattern. The distance of HRP labeled and unlabeled fields from the midline was then measured along the length of the dorsal and ventral halves of lobule VIII. These measurements were then used to reconstruct the overall pattern of labeling using ClarisDraw.

\section{RESULTS}

\section{En-2 expression has no effect on L7- $\beta$-gal sagittal boundaries}

To examine the effects of ectopic En-2 expression on late embryonic and early postnatal cerebellar patterning, transgenic mouse lines (L7En-2 mice) were made in which En-2 protein was specifically expressed in Purkinje cells using the $p c p$-2(L7) promoter (Baader et al., 1998). One rationale for this experiment was the apparent complementarity of en2 and pcp-2(L7) gene expression patterns based on previous descriptions of the late embryonic pattern of En-2 expression (Millen et al., 1995). Thus, expression of En-2 from the $p c p-2(L 7)$ gene promoter would be expected to upset the normal sagittal pattern of En-2 during late embryogenesis by forcing expression in normally En-2-negative regions within the layer of developing Purkinje cells, and this was actually observed (Fig. 1) (Baader et al., 1998). However, because the primary patterning event that gives rise to both mediolateral and rostrocaudal boundaries in the cerebellum appears to occur between E9 and E14.5 (Herrup and Kuemerle, 1997; Baader et al., 1998), and $p c p$-2(L7) gene expression is initiated only after E14 (Smeyne et al., 1991; Oberdick et al., 1993), ectopic expression of En-2 from the $p c p-2(L 7)$ promoter was predicted to have no effect on formation of cerebellar compartments.

As predicted, sagittal patterning in L7En-2 transgenics is normal from the neonatal period (Fig. $2 A, B$ ) to the adult stage ( 3 months) (Fig. 2C,D), despite the obvious decrease in cerebellar size in the adult animals. This was demonstrated by crossing L7En-2 mice into a transgenic mouse background in which sagittal zones are revealed by expression of an L7- $\beta$-gal fusion protein (Oberdick et al., 1993). One advantage of this $\beta$-gal fusion gene is that, in contrast to the endogenous L7 gene and transgenes carrying longer promoter fragments that show uniform expression in adults, this one reveals a banding pattern that persists into adult stages. In general, L7- $\beta$-gal band widths appear approximately equivalent in the wild-type and L7En-2 mice, which is visible in both whole-mount (Fig. 2) and coronal sections (data not shown).

There is some tendency, however, for selected bands near to the midline to show some decrease in width. For example, the broad L7- $\beta$-gal-positive zone nearest the midline in lobules VI and VII, and the L7- $\beta$-gal-negative zone at the midline in lobule VI, are reduced in width $(\sim 35 \%)$ by a factor greater than the overall decrease in the mediolateral dimension of the cerebellum $(\sim 12 \%)$. In contrast, L7- $\beta$-gal band widths and spacing in lobule

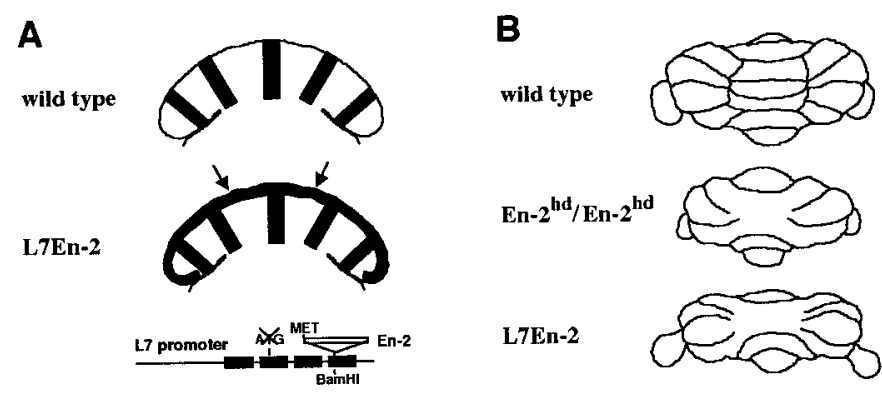

Figure 1. Schematics showing effects on En-2 expression pattern and cerebellar morphology in L7En-2 transgenic mice. $A$, The patterns of En-2 expression in E17.5 cerebellum of wild-type and L7En-2 transgenics were determined by in situ hybridization and compared. Drawings are in the horizontal plane. The pattern in L7En-2 transgenics is a fusion of transgene and endogenous En-2 expression patterns, resulting in a more uniform pattern of En-2-encoding transcripts than in wild types. Drawings of expression patterns and L7En-2 gene construct are adapted from data presented by Baader et al. (1998) and Millen et al. (1995). It should be noted that expression of En-2 in normal embryonic mouse cerebellum is not restricted to Purkinje cells and includes EGL precursors and immature deep nuclear cells, whereas transgene-driven En-2 expression is restricted to the Purkinje cell cortical region (arrows). B, Distribution of morphological defects observed in adult En $2^{\text {hd }} / E n 2^{\text {hd }}$ homozygous null mutants versus L7En-2 transgenic mutants. The homozygous null animals have a larger reduction in the mediolateral axis; L7En-2 transgenics are more severely affected in the rostrocaudal and dorsoventral axes. These relative changes were confirmed by direct comparison of sections that were used to prepare Figure 7. 

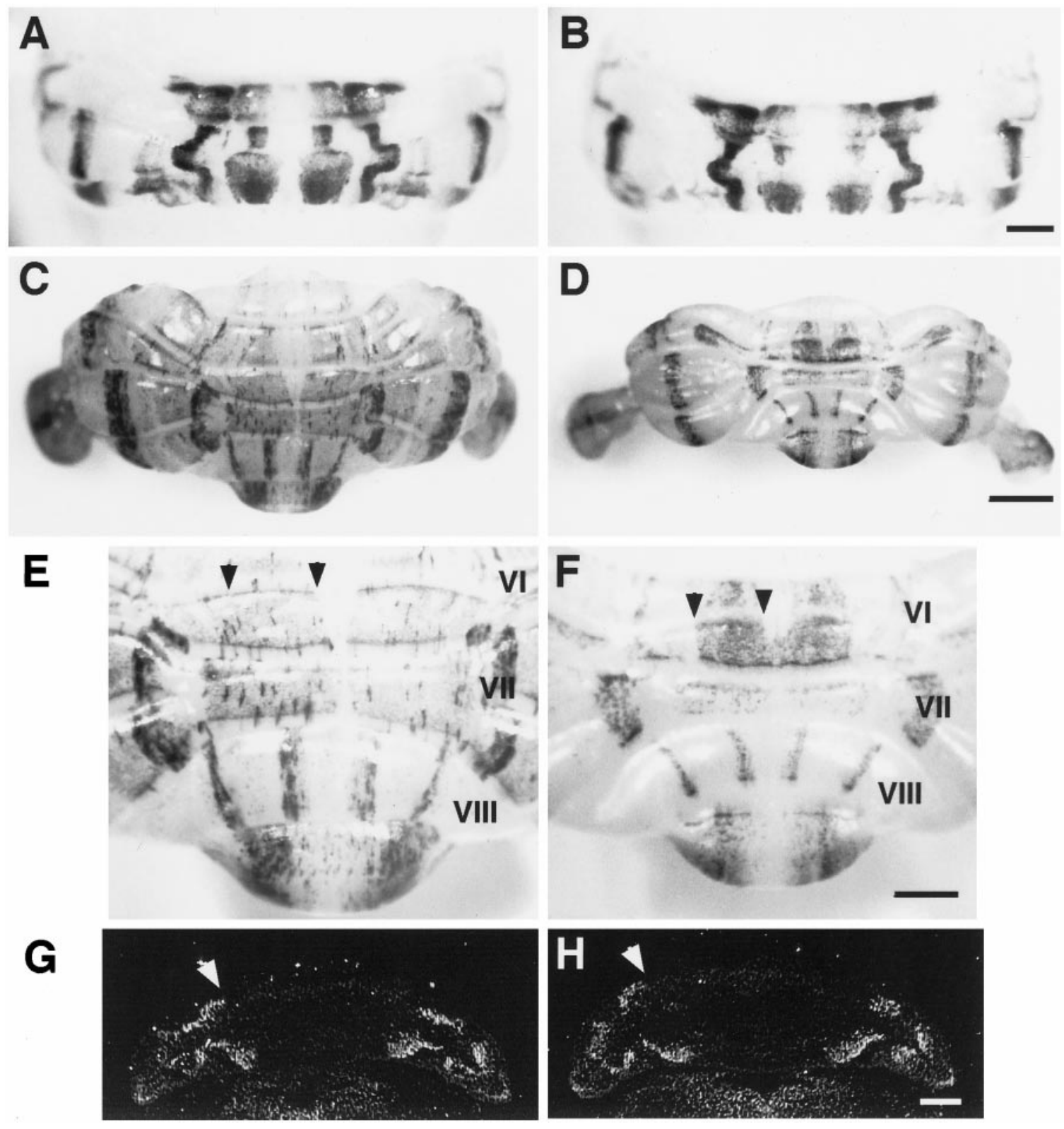

Figure 2. Lack of effect on sagittal organization within the cerebellar cortex. $A-F$, Whole-mount views of wild-type $(A, C, E)$ and L7En-2 transgenic $(B, D, F)$ cerebella stained for L7- $\beta$-gal expression. Heterozygous L7En-2 transgenic animals were crossed to heterozygous L7 $\beta$ G3 mice (Oberdick et al., 1993), and positives for both transgenes were identified by PCR. The wild-type and mutant pair in each set are littermates. P0 ( $A, B), 3$ month $(C$, $D$ ), and high magnification to reveal lobule VIII $(E, F)$. Note that, in general, most of the change that occurs in the mutant occurs after P0 and mainly affects the rostrocaudal axis (rostral is top, and caudal is bottom in each image). $G, H$, In situ hybridization with probe to cadherin-8. The banding pattern in neonatal wild-type mice $(G)$ is identical to that in L7En-2 mutants $(H)$. Scale bars: $A, B, 0.5 \mathrm{~mm} ; C, D, 1 \mathrm{~mm} ; E-H, 0.5 \mathrm{~mm}$. White arrowheads in $G$ and $H$ indicate borders of Cad-8 expression; black arrowheads in $E$ and $F$ indicate the near-midline L7- $\beta$-gal zone that is decreased in width in L7En-2.

VIII are relatively unaffected. Although regional differences in effects on sagittal zones cannot be excluded, and it is quite clear that there are in fact selective effects that occur within the fissures (Baader et al., 1998), the general pattern of compartmentation as revealed by L7- $\beta$-gal is remarkably normal in L7En- 2 mice.

The $\mathrm{Ca}^{2+}$-dependent cell adhesion protein cadherin- 8 has been shown to be expressed in neonates in a restricted sagittal pattern (Arndt et al., 1998). Because of the potential role of this gene in controlling formation of cellular clusters in the developing cerebellum, its pattern of expression was examined in L7En-2 transgenics. Like L7- $\beta$-gal, the pattern of Cad-8 was not affected in neonatal mutants (Fig. $2 G, H$ ).
Thus, with respect to early onset sagittal banding patterns, there is little to distinguish the phenotype of the En-2 overexpressor from that of null mutants, because the latter, too, have only subtle effects on sagittal banding revealed using the L7- $\beta$-gal marker (Kuemerle et al., 1997). In addition, Purkinje cell numbers in both mutants are decreased by a similar amount, by $\sim 40 \%$. However, whereas the loss in En2 ${ }^{\text {hd }}$ null mutants was suggested to be an effect on the early generation of Purkinje cells, that in L7En-2 overexpressor mice is most likely a result of postmitotic effects (Kuemerle et al., 1997; Baader et al., 1998). Different mechanisms of loss are suggested by the fact that, despite the similar quantitative level of cell loss, the effect on 
global cerebellar morphogenesis is quite distinct. Specifically, En-2 overexpressors show much less of a reduction in the mediolateral axis than do En $2^{\text {hd }}$ null mutants but a larger decrease in both the rostrocaudal and dorsoventral axes (Fig. $1 B$ ). To determine whether these two mutants could be distinguished by other criteria, the analysis of more sagittal markers was undertaken.

\section{Ectopic En-2 expression disrupts zebrin II, P-Path, and NADPH-diaphorase sagittal boundaries}

To better characterize the effect of ectopic En-2 expression on sagittal compartmentation, several other molecular patterns were examined. Zebrin II (or aldolase C) (Ahn et al., 1994) is a Purkinje cell marker expressed during early postnatal cerebellar development in a uniform pattern that gives way to stripes during the third and fourth postnatal weeks by a process of selective downregulation in discrete zones (Leclerc et al., 1988). In an earlier study of $E n 2^{\text {hd }}$ null mutants, the zebrin II banding pattern was found to differ in subtle ways from that in wild types, but like the L7- $\beta$-gal pattern, it was found to be normal overall (Kuemerle et al., 1997). In contrast, ectopic overexpression of En-2 resulted in gross perturbation of the zebrin II sagittal pattern in L7En-2 mice $(n=4)$ (Fig. $3 A)$. Although evidence of sagittal banding remains, the bands appear fractured, and the boundaries between zones appear diffuse. These effects can be seen throughout the rostrocaudal extent of the cerebellum and are particularly evident in lobules VIII and IX in the caudal portion of the cerebellum (Figs. $3 A, 4$ ) and throughout the anterior lobe (Fig. 5A-D). Generally, there is a tendency for caudal regions to be more severely affected than rostral ones.

There is a $40 \%$ decrease in the overall level of zebrin II expression in L7En-2 mice as measured by Western blot analysis, but this is equivalent to the decrease observed for all Purkinje cell markers, including L7 and calbindin (Sanlioglu et al., 1998). Thus, this decrease is assumed to be caused by a relative decrease in the numbers of Purkinje cells. Rather than a selective loss of zebrin II-negative or -positive Purkinje cells, therefore, the change observed here is clearly a redistribution of expression, resulting in a breakdown in the normal precision of sagittal patterning. As is shown in Figure 4, $C$ and $D$, the diff usion of boundaries appears to be a result of randomized upregulation and downregulation of zebrin II such that positive cells appear in normally negative zones and vice versa.

Although the effect on the zebrin II banding pattern is grossly different from that on L7- $\beta$-gal, like L7- $\beta$-gal, there are some detectable heterogeneities, e.g., the diffusion of zebrin II boundaries is more prominent in lobule VIII than in the anterior lobe, and in the anterior lobe, there is a slight gradient of increasing severity in the rostral to caudal direction (Fig. 5). In addition, in some regions, there are zebrin II bands in L7En-2 mice that are still identifiable with their wild-type counterparts, and effects on widths and spacing of these bands seem to vary along rostrocaudal divisions. Relative to wild-type mice, for example, in L7En-2 mice, the spacing of zebrin II bands near the midline in the anterior lobe is decreased by an even greater degree $(50 \%)$ than are the L7 band widths and spacing in the more caudal lobules VI and VII (Figs. 2, 6). Combined with the lack of change in L7- $\beta$-gal band spacing in lobule VIII (Fig. 2), this suggests a caudal to rostral increase in the spacing effect. This is opposite to the border diffusion effect on late onset banding, which seems most severely affected in lobule VIII. To compound this complexity, the effect on zebrin II patterning is greatest deep within lobule VIII compared with at the apex.
A

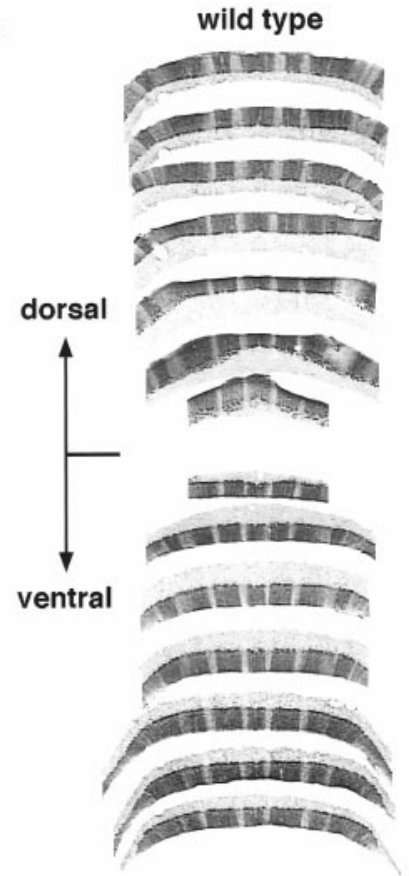

B

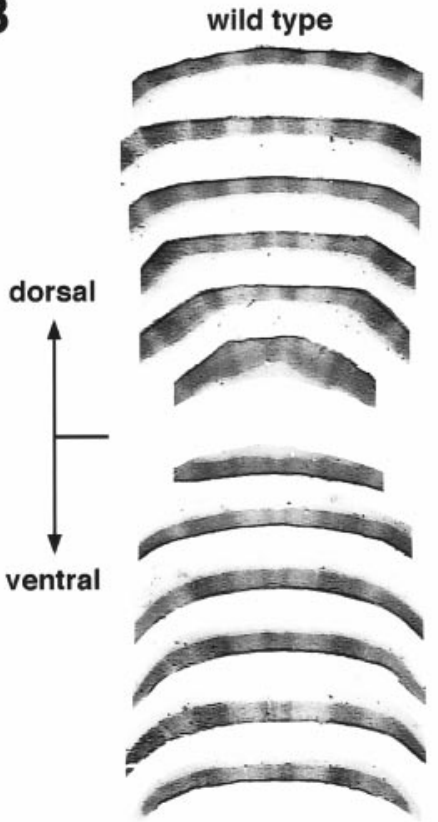

L7En-2
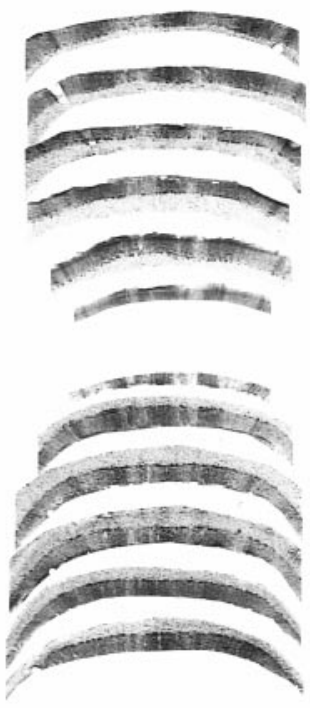

L7En-2
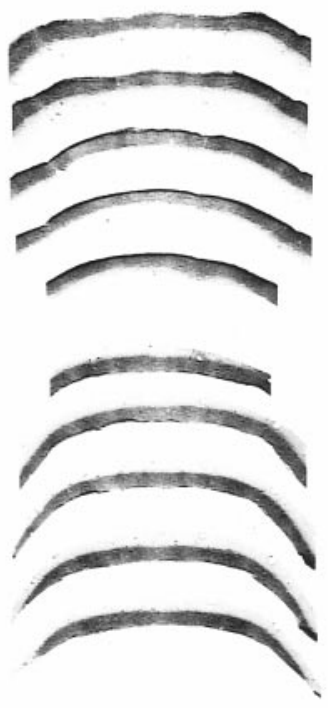

Figure 3. Effect of ectopic En-2 expression on zebrin II and P-Path sagittal banding in lobule VIII. $A$, Reconstruction of zebrin II banding pattern in lobule VIII prepared from semi-adjacent coronal sections. The dorsal and ventral portions of the lobule were separated and graphically processed to produce a "fillet" of the lobule (see Materials and Methods). Note the severe disruption of banding in the L7En-2 transgenic. $B$, Reconstruction of P-Path banding pattern in lobule VIII prepared from semi-adjacent coronal sections processed as in $A$. Note the severe disruption of banding in the L7En-2 transgenic.

The diffusion effect observed for zebrin II was confirmed by examining the pattern of expression of another late onset sagittal marker, P-Path (Leclerc et al., 1992). Zebrin II has an approximately complementary staining pattern to P-Path, and similar to 

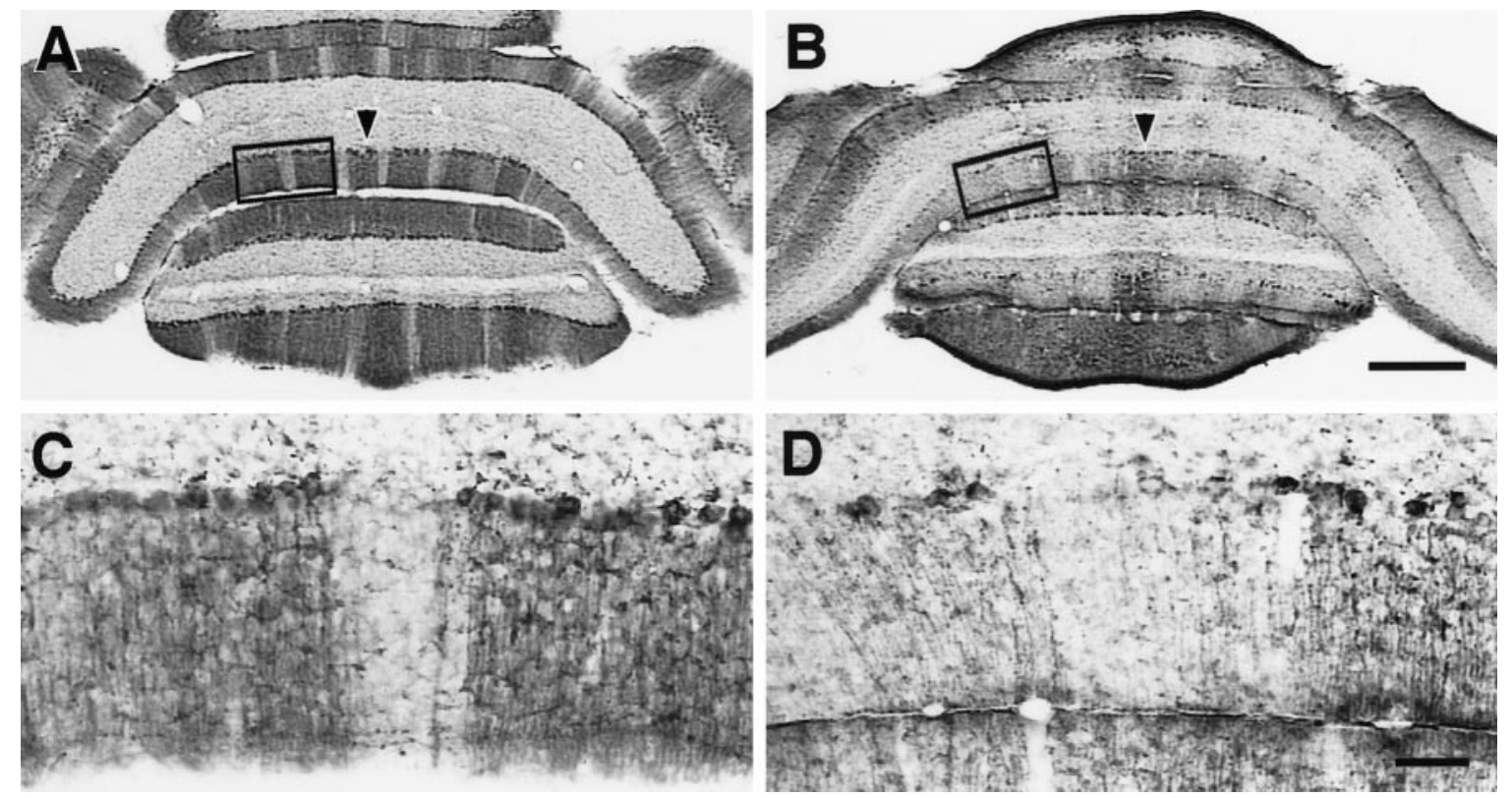

Figure 4. Detailed view of unpatterned zebrin II expression in caudal cerebellum of L7En-2 mutants. $A$, $B$, Coronal sections through caudal adult cerebellum were stained for zebrin II protein. Zebrin II in lobule V III of normal mice is expressed in a midline band (arrowhead) and three bilaterally symmetric bands that are spaced by sharply defined negative zones $(A)$. Expression in L7En-2 transgenics is concentrated in three broad bands with poorly defined boundaries $(B)$. This can also be seen in the reconstruction of Figure 3. $C, D$, Higher-magnification views of boxed regions in $A$ and $B$ showing sharp zebrin II boundaries in the wild-type $(C)$ and randomization of zebrin II-positive and -negative cells in L7En-2 mutants $(D)$. Scale bars: $A, B, 0.5 \mathrm{~mm} ; C, D, 0.1 \mathrm{~mm}$

the changes in zebrin II expression, the pattern of P-Path bands was disrupted in L7En-2 transgenics $(n=3)$ (Fig. $3 B)$. The normal boundaries of P-Path expression become diffuse in the mutant, and the normal precision of sagittal banding is compromised. The same effect was observed with a third Purkinje cell marker called zebrin I (Hawkes et al., 1985), which reveals bands identical to zebrin II (data not shown).

One possibility is that the pattern of zebrin II expression is regulated by a different set of cues than those regulating L7 expression. Because the boundaries defined by zebrin II expression appear to be coincident to a large degree with those respected by both climbing and mossy fiber afferents (Gravel et al., 1987; Gravel and Hawkes, 1990), one possibility is that zebrin II expression is regulated extrinsically by one or both of these systems, which are themselves perturbed in L7En-2 mice. To begin to approach this issue, another molecular compartmental pattern was examined. NADPH-diaphorase, a histochemical marker for nitric oxide synthase (NOS), which is selectively expressed in granule cells and basket/stellate cells of the cerebellum, is regulated in granule cell cultures by excitatory neurotransmission (Baader and Schilling, 1996). In the granule cell layer of several lobules, particularly lobule VI, diaphorase is expressed in a pattern of "blobs" oriented in the sagittal plane; these highly reproducible stereotypical patterns have been suggested to be linked to mossy fiber activity and arrangement (Hawkes and Turner, 1994; Schilling et al., 1994). Like the zebrin II banding pattern, which is visible as well in this area and shows the typical diff usion in the mutant (Fig. $5 A-D$ ), the pattern of NOS modules in lobule VI/VII essentially disappears $(n=3)$ (Fig. $5 E, F)$.

\section{Disruption of spinocerebellar mossy fiber projection patterns}

Based on previous reports (Hawkes and Turner, 1994; Schilling et al., 1994), one explanation for the effect on NOS patterning is that ectopic En-2 expression perturbs the organization of mossy fiber projections. To test this possibility and to provide some morphological indication of effects on sagittal organization, wheat germagglutinin injections were made into the rostral end of the lumbar spinal cord of wild-type and L7En-2 littermates. In normal mice, anterograde transport of the label typically resolves a series of positive and negative zones in lobule VIII and in the anterior lobe (Arsénio-Nunes and Sotelo, 1985; Vogel et al., 1996). This pattern was found to be perturbed in L7En-2 mice (Fig. 6A-F). In particular, rather than three clearly defined negative zones and four positive zones distributed throughout lobule VIII, there is one broad negative zone fixed at the apex of the lobule. The pattern throughout both the dorsal and ventral halves of lobule VIII was reconstructed for both wild-type and L7En-2 mutant littermates (Fig. $7 A, B$ ). As can be seen, throughout much of lobule VIII of the transgenic, the mossy fiber distribution is uniform with only thin wisps of negative zones. The effects in the anterior lobe appear generally less severe than those in lobule VIII in the sense that the basic compartmental nature of the mossy fiber pattern can still be resolved (Fig. $6 G-M$ ), and this general gradient of effect is similar to that observed for zebrin II banding. Despite the lesser severity in the anterior lobe, the boundaries between positive and negative mossy fiber zones appear diff use, again paralleling the effect on zebrin II. Opposite to the effect on zebrin II, however, the effect on mossy fiber distribution is most severe at the apex of lobule VIII than at the base (Fig. 6A-F).

In contrast to the case in L7En-2 transgenics, the organization of spinocerebellar mossy fibers in $E n-2^{\text {hd }}$ null mutant mice is minimally affected as shown in Figure 7, $C$ and $D$ (Vogel et al., 1996). This is of interest because the $E n-2^{\text {hd }}$ null mutant was characterized by loss of $\sim 40 \%$ of the Purkinje cells and a proportional loss of other cerebellar neurons, which is nearly iden- 

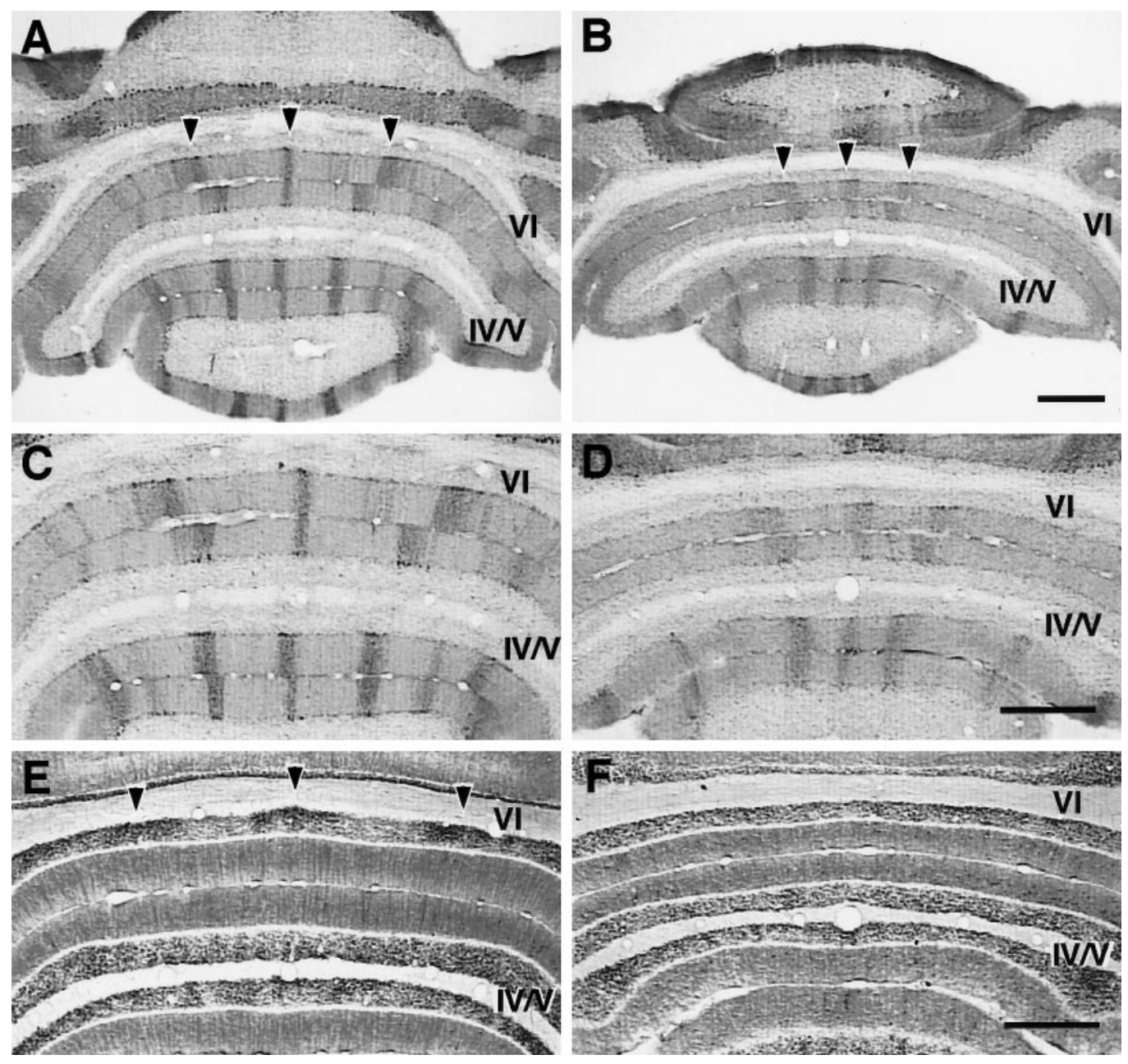

Figure 5. Disruption of Purkinje cell and granule cell patterning in lobule VI and anterior lobe of L7En-2 mutants. In horizontal sections through lobule VI and the anterior lobe, zebrin II is expressed in sharply defined zones $(A)$, the borders of which are diffuse in L7En-2 transgenics $(B)$. This is shown at higher magnification in $C$ and $D$. The diff usion effect is strongest in more caudal regions, such as lobule VI (arrowheads in $A$ and $B$ ). $E, F$, Adjacent sections to those shown in $C$ and $D$ stained for NADPH-diaphorase. In the adult wild type $(E)$, three major bands in the granule cell layer are identified by this marker (arrowheads in $E$ ). These bands are undetectable in L7En-2 mutants $(F)$. The data shown in this figure were confirmed in three pairs of animals. Scale bars: $A-F, 0.5 \mathrm{~mm}$.

tical to the extent of cell loss in L7En-2 mice (Kuemerle et al., 1997; Baader et al., 1998). In addition, the size of the cerebellum in the mediolateral dimension is more severely affected in En-2 ${ }^{\text {hd }}$ null mutants.

\section{DISCUSSION}

Here, we provide evidence that an En-2-sensitive property of Purkinje cells selectively affects late onset sagittal banding patterns. In parallel with this, at least one morphological indicator of sagittal compartments, mossy fiber projections, is also disrupted. In particular, it is demonstrated that the basic sagittal arrangement of Purkinje cells is unaffected in L7En-2 mice as revealed by expression of L7- $\beta$-gal and cadherin-8, providing a fundamental substructure against which effects on late onset patterns (zebrins, P-Path, NADPH-diaphorase) and on mossy fibers could be compared. The questions that arise are as follows: (1) what is the correlation between the En-2 expression pattern in L7En-2 animals and the observed phenotype with respect to sagittal banding?; and (2) based on the En-2 expression pattern and the demonstrated phenotype, what can be concluded about the mech- anisms that are involved in the changes of patterning observed in cerebella of L7En-2 mice?

\section{Relationship between En-2 expression and effects on banding patterns}

Endogenous En-1 and En-2 are expressed during the earliest stages of formation of the cerebellar primordium, approximately E9 (Davis and Joyner, 1988), and the defined expression domain is certain to include Purkinje cell precursors. This is supported by the loss of the entire cerebellar primordium in En-1 null mutants (Wurst et al., 1994). At E17.5, En-1 and En-2 are both expressed in a pattern of sagittally oriented bands (Millen et al., 1995). In L7En-2 transgenic mice, however, the pattern of endogenous En-2 expression characteristic of the wild type is overwhelmed by transgene expression, which fills in the normally En-2-negative zones (Baader et al., 1998). In addition, from this time onward, expression of En-2 in Purkinje cells of L7En-2 mice persists into adulthood, whereas endogenous En-2 expression normally becomes confined to the external germinal layer (EGL) and granule cells postnatally (Davis and Joyner, 1988).

Despite both aspects of ectopic expression, with respect to 

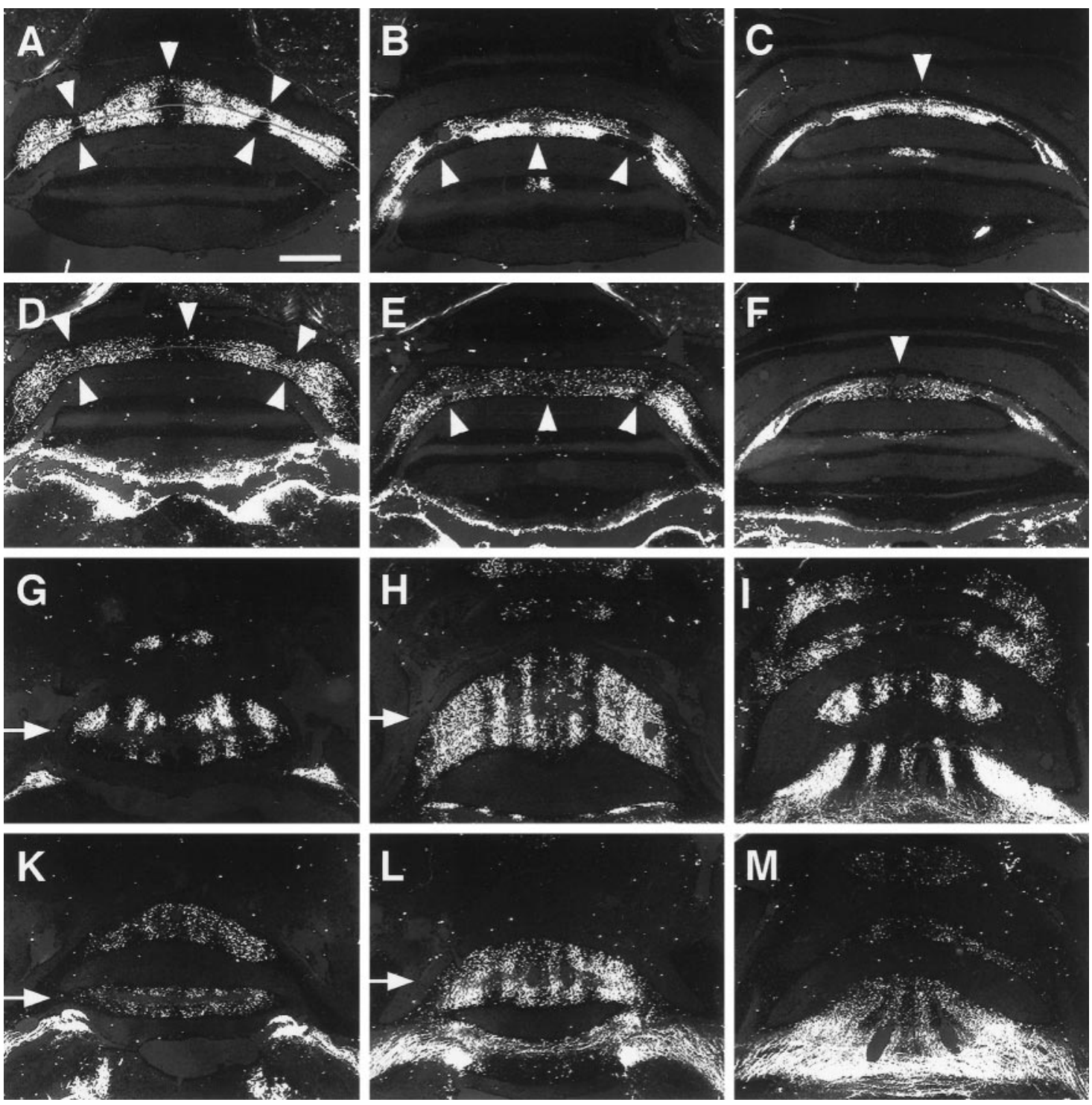

Figure 6. Effect of ectopic En-2 expression on sagittal organization of spinocerebellar mossy fiber projections. $A-C$, Caudal to rostral series of coronal sections through lobule VIII in wild types. Spinocerebellar mossy fibers resolve into a series of sharply defined positive and negative zones. $D-F$, Caudal to rostral series of coronal sections through lobule VIII of L7En-2s. There is some evidence of patterning that is mainly visible at the midline, but the pattern is much more diff use and some negative zones appear to be absent. Arrowheads in $A-F$ mark the positions of zones that are aberrant in L7En-2s. $G-I$, Dorsal to ventral series of horizontal sections through the anterior lobe of wild types. Spinocerebellar mossy fibers reveal a series of sagittal bands interrupted by zones that do not receive these projections. $K-M$, Dorsal to ventral series of horizontal sections through the anterior lobe of L7En-2s. A pattern similar to that in wild types is observed, but the distribution of fibers is much more diff use. The observations presented here are representative of nine wild-type cases and 12 transgenic cases prepared from two litters. Note that the bright positive regions in the brainstem (bottom) of $D-F$ and $K-M$ are fibers of passage. Scale bar: $A-M, 0.5 \mathrm{~mm}$.

sagittal banding embryonically and cell type postnatally, there is surprisingly little correlation between the pattern of transgene expression and the developmental defects observed. For example, it has been shown previously that expression of a toxin protein using the same promoter as used in this study resulted in vermal Purkinje cell loss beginning in the neonatal period, followed by protracted loss of cells in the hemispheres (Smeyne et al., 1995), in keeping with the general medial to lateral spread of expression of both endogenous L7 and L7-based transgene expression (Oberdick et al., 1993). Evidence of cell loss restricted to sagittal zones was even reported in the earlier toxin study. Such is clearly not the case in L7En-2 mice. Thus, rather than reflect a one-forone match between transgene expression and phenotype, some subset of cerebellar cells not obviously related to the pattern of transgene expression is selectively sensitive to ectopic En-2 expression. This conclusion is based mainly on the observation of intact banding patterns of L7- $\beta$-gal and cadherin- 8 . The simplest explanation for this is that the $>30 \%$ loss of Purkinje cells is distributed approximately equally across the mediolateral axis of the L7En-2 cerebellum.

Whatever the mechanism for the selective effect on late onset patterns, one thing that is clear is that it is not uniformly distributed. For example, the diff usion of zebrin II stripe boundaries in L7En-2 is greatest in caudal cerebellar regions, especially lobule VIII, but less severe in the anterior lobe. Similarly, despite a general lack of effect on both L7- $\beta$-gal and zebrin banding in $E n-2^{\text {hd }}$ null mice, some highly localized subtleties were observed (Kuemerle et al., 1997). Two examples of this that are most 
A
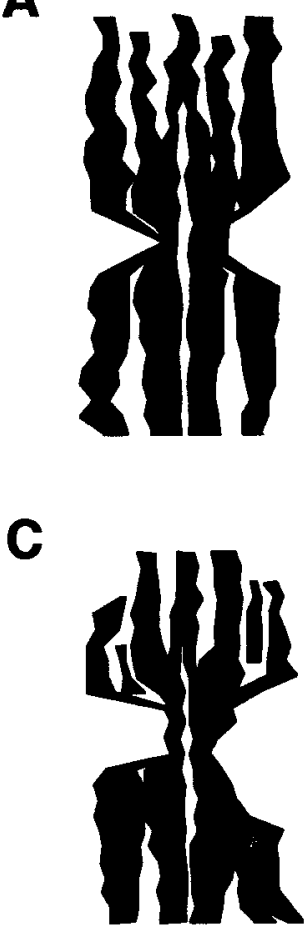

B
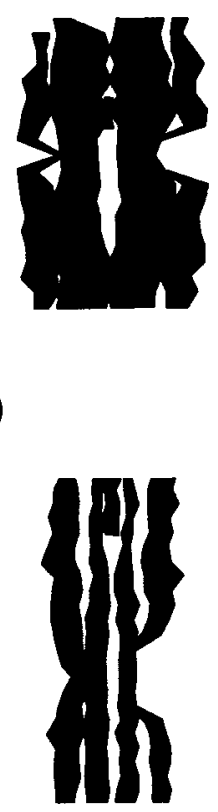

$0.2 \mathrm{~mm}$
$1 \mathrm{~mm}$

Figure 7. Two-dimensional reconstruction of spinocerebellar mossy fiber projections to lobule VIII: comparison of pattern in L7En-2 transgenics with that in $E n 2^{\text {hd }} / E n 2^{\text {hd }}$ homozygous null animals. $A, C$, In the wild type, mossy fibers from rostral lumbar spinal cord segregate into four well defined projection zones (black) in the ventral half of lobule VIII; in the dorsal half of the lobule, the same bands are apparent but broken into multiple smaller bands. These positive zones are separated by narrow zones that do not receive innervation from this spinal cord region. A thin negative zone extends throughout most of the midline. $B$, In L7En-2 transgenics, a broad negative zone distinguishes the midline, but it is focused at the apex of lobule VIII. In addition, the lateral negative zones are primarily missing. The overall result is an effective fusion of projection zones such that, at any point in the lobule, the typical heterogeneity observed in the wild-type is absent. $D$, The pattern of mossy fiber segregation is mostly intact in homozygous $E n 2^{\text {hd }} / E n 2^{\text {hd }}$ mutants. Note that the width of lobule VIII of L7En-2 transgenics $(B)$ is less severely affected than that of null mutants $(D)$; nevertheless, mediolateral patterning is more severely affected in L7En-2 overexpressors.

relevant to the current study were the insertion of L7- $\beta$-galpositive bands in lobules VIII and IX with no noticeable effects on zebrin bands. In contrast, we have reported previously the deletion of one L7- $\beta$-gal band in lobule IX of L7En-2 mice (Baader et al., 1998). Thus, the combined observations from the loss-of-function and gain-of-function approaches indicates that En-2 may play a role in restricting L7 expression in a complex, heterogeneously distributed manner. In fact, negative regulation of the $p c p-2(L 7)$ promoter by En-2 was recently reported (Sanlioglu et al., 1998).

\section{Cell loss as a possible mechanism of pattern disruption}

One confounding aspect to the interpretation of these data are the loss of $>30 \%$ of the Purkinje cells and a secondary loss of other cerebellar cells (Baader et al., 1998). However, there is little evidence to suggest that the diffusion of late onset boundaries could be caused by sagittally restricted cell loss. On the other hand, another effect, decrease in the width and spacing of some L7- $\beta$-gal and zebrin II bands that are still identifiable with their wild-type counterparts, may be explained by a selective tendency of near-midline cerebellar regions toward cell loss. If so, then this is important because the latter effect is most severe in rostral regions, whereas the major effect on boundary diffusion is most severe caudally. These observations support the view that the diff usion of late onset boundaries is unrelated to cell loss.

With respect to the organization of afferents, the simplest explanation for the redistribution of spinocerebellar mossy fibers in L7En-2 mice might be the decrease in size of the available target pool, which would force normally segregated axons to seek out new territories. In fact, there are hints that ablation of external granule layer cells and the cell loss associated with spontaneous cerebellar mutants, such as staggerer, leads to a disruption of mossy fiber topography (Arsénio-Nunes et al., 1988; Ji and Hawkes, 1996). If this target size model was correct, then the same redistribution of mossy fibers should have been observed in En-2 ${ }^{\text {hd }}$ null mice because the latter are characterized by an equivalent degree of cell loss as observed in L7En-2 mice (Kuemerle et al., 1997). As reported here, such a redistribution is not observed. In addition, Purkinje cell loss in Lurcher mutants, in staggerer mutants studied at an earlier age than in the work cited above, and granule cell degeneration in a model of parvovirus-induced cerebellar hypoplasia showed only minor or no influence on the positioning of mossy fiber terminals (OsterGranite and Herndon, 1976; Vogel and Prittie, 1994; Ji et al., 1997). Therefore, the relatively limited cell loss and lack of morphological defects in L7En-2 mice would seem to exclude from consideration effects on cell viability to account for the degree of change observed in mossy fiber projection patterns and late onset banding patterns.

\section{Mechanisms of Engrailed action with respect to sagittal organization}

It is impossible at this time to impose a defined model on the observations reported here. Most likely, the effect of En-2 expression on late onset banding patterns and afferent projections is linked to molecular changes that are induced (or repressed) in the surviving cerebellar cells. The clearest conclusion that can be drawn is that there is an En-2-sensitive property of Purkinje cells that is critically important for coherent banding patterns of the late type, and for the precise recognition of boundaries by mossy fibers. It is possible that direct interactions of mossy fibers with guidance cues on Purkinje cells, through transient contacts or collaterals, could be important for the normal sagittal arrangement of mossy fibers (Arsénio-Nunes and Sotelo, 1985; ArsénioNunes et al., 1988). In fact, Engrailed has been demonstrated to be involved in axonal patterning both in Drosophila and vertebrates, ostensibly via the regulation of guidance or adhesion molecules (Friedman and O'Leary, 1996; Itasaki et al., 1996; Rétaux et al., 1996; Siegler and Jia, 1999). Perturbation of the expression of similar cues in L7En-2 mice might result in the disruption of mossy fiber projection patterns, thereby affecting the expression of NOS or other proteins within the granule cell layer (Schilling et al., 1994; Baader and Schilling, 1996), which might in turn induce, via parallel fibers, changes in the expression of other late onset markers. Although contradictory to experiments suggesting intrinsic developmental control of zebrin banding (Wassef et al., 1990; Seil et al., 1995), such a hierarchy cannot be excluded 
because all such reports describe evidence of zebrin-positive and -negative clusters of Purkinje cells without direct reference to or comparison with wild-type zebrin banding patterns.

Alternatively, the positioning and development of granule cells or their activity could be a critical step in refining and/or stabilizing sagittal banding patterns. In fact, activity-dependent and growth factor-dependent mechanisms are known to play a role in organizing axonal projections (Goodman and Schatz, 1993; Catalano and Schatz, 1998; Penn et al., 1998). An activity-dependent mechanism might also explain why some semblance of late onset sagittal order is still observed, and likewise why mossy fiber projection territories are generally similar (albeit broadened) to wild-type ones in L7En-2 mutants. For example, such a mechanism may be critical for maintenance or refinement of pattern as opposed to initial formation.

Last, the late onset patterns may be dictated by an intrinsic En-2-sensitive mechanism distinct from that directing early onset patterns, and the arrangement of mossy fibers may be influenced by this late onset mechanism. That both early and late onset patterns might be determined by intrinsic mechanisms has been suggested based on transplantation and cell culture experiments (Wassef et al., 1990; Oberdick et al., 1993; Seil et al., 1995; Chédotal et al., 1996). A variant of this model might be a patterning process that was divided into multiple phases. In fact, a "double patterning" hypothesis has been proposed previously to account for patterning events that occur before E14.5 (Kuemerle et al., 1997). In the context of this hypothesis, the data described herein support either a third patterning event or a much more prolonged second event with distinct phases of its own (e.g., initiation, maintenance, refinement, etc.).

One major step on the way to distinguishing these several possibilities will be to analyze the organization of the climbing fibers. If the topography of the latter projections is found to be normal, for example, this might suggest two independent boundary systems involved in organizing afferent projections. That mossy fiber and climbing fiber projections are inherently different is suggested by differences in the nature of their targets and in the timing of their sagittal organization. This could also be shown in functional terms by comparison of climbing fiber versus mossy fiber cutaneous receptive field maps projecting to the cat $\mathrm{C} 3$ zone; mossy fiber receptive fields showed significant overlap, whereas those of climbing fibers did not (Garwicz et al., 1998). This would suggest an intrinsically greater degree of accuracy of projection within the climbing fiber population compared with mossy fibers, and therefore might predict some differences in behavior of the two fiber systems in the L7En-2 mouse.

\section{REFERENCES}

Ahn AH, Dziennis S, Hawkes R, Herrup K (1994) The cloning of zebrin II reveals its identity with aldolase C. Development 120:2081-2090.

Arndt K, Nakagawa S, Takeichi M, Redies C (1998) Cadherin-defined segments and parasagittal cell ribbons in the developing chicken cerebellum. Mol Cell Neurosci 10:211-228.

Arsénio-Nunes ML, Sotelo C (1985) Development of the spinocerebellar system in the postnatal rat. J Comp Neurol 237:291-306.

Arsénio-Nunes ML, Sotelo C, Wehrlé R (1988) Organization of spinocerebellar projection map in three types of agranular cerebellum: Purkinje cells vs. granule cells as organizer element. J Comp Neurol 273:120-136.

Baader SL, Schilling K (1996) Glutamate receptors mediate dynamic regulation of nitric oxide synthase expression in cerebellar granule cells. J Neurosci 16:1440-1449.

Baader SL, Sanlioglu S, Berrebi AS, Parker-Thornburg J, Oberdick J (1998) Ectopic overexpression of Engrailed-2 in cerebellar Purkinje cells causes restricted cell loss and retarded external germinal layer development at lobule junctions. J Neurosci 18:1763-1773.

Bian F, Chu T, Schilling K, Oberdick J (1996) Differential mRNA transport and the regulation of protein synthesis: selective sensitivity of Purkinje cell dendritic mRNAs to translational inhibition. Mol Cell Neurosci 7:116-133.

Brochu G, Maler L, Hawkes R (1990) Zebrin II: a polypeptide antigen expressed selectively by Purkinje cells reveals compartments in rat and fish cerebellum. J Comp Neurol 291:538-552.

Catalano SM, Shatz CJ (1998) Activity-dependent cortical target selection by thalamic axons. Science 281:559-562.

Chédotal A, Pouquié O, Ezan F, San Clemente H, Sotelo C (1996) BEN as a presumptive target recognition molecule during the development of the olivocerebellar system. J Neurosci 16:3296-3310.

Davis CA, Joyner AL (1988) Expression patterns of the homeoboxcontaining genes En-1 and En-2 and the proto-oncogene int-1 diverge during mouse development. Genes Dev 2:1736-1744.

DeOlmos J, Hardy H, Heimer L (1978) The afferent connections of the main and the accessory olfactory bulb formations in the rat: an experimental HRP study. J Comp Neurol 181:213-244.

Friedman GC, O'Leary DDM (1996) Retroviral misexpression of engrailed genes in the chick optic tectum perturbs the topographic targeting of retinal axons. J Neurosci 16:5498-5509.

Garwicz M, Jörntell H, Ekerot C-F (1998) Cutaneous receptive fields and topography of mossy fibres and climbing fibres projecting to cat cerebellar C3 zone. J Physiol (London) 512:277-293.

Goodman CS, Shatz CJ (1993) Developmental mechanisms that generate precise patterns of neuronal connectivity. Cell 72:77-98.

Gravel C and Hawkes R (1990) Parasagittal organization of the rat cerebellar cortex: direct comparison of Purkinje cell compartments and the organization of the spinocerebellar projection. J. Comp. Neurol. 291:79-102.

Gravel C, Eisenman LM, Sasseville R, Hawkes R (1987) Parasagittal organization of the rat cerebellar cortex: direct correlation between antigenic Purkinje cell bands revealed by mabQ113 and the organization of the olivocerebellar projection. J Comp Neurol 265:294-310.

Hawkes R, Turner RW (1994) Compartmentation of NADPHdiaphorase activity in the mouse cerebellar cortex. J Comp Neurol 346:499-516.

Hawkes R, Colonnier M, Leclerc N (1985) Monoclonal antibodies reveal sagittal banding in the rodent cerebellar cortex. Brain Res 333:359-365.

Herrup K, Kuemerle B (1997) The compartmentalization of the mouse cerebellum. Annu Rev Neurosci 20:61-90.

Itasaki N, Nakamura H (1996) A role for gradient En expression in positional specification on the optic tectum. Neuron 16:55-62.

Ji ZQ, Hawkes R (1996) Partial ablation of the neonatal external granular layer disrupts mossy fiber topography in the adult rat cerebellum. J Comp Neurol 371:578-588.

Ji ZQ, Jin Q, Vogel MW (1997) Evidence of spinocerebellar mossy fiber segregation in the juvenile Staggerer cerebellum. J Comp Neurol 378:354-362.

Korematsu K, Redies C (1997) Expression of cadherin-8 mRNA in the developing mouse central nervous system. J Comp Neurol 387:291-306.

Kuemerle B, Zanjani H, Joyner A, Herrup K (1997) Pattern deformities and cell loss in Engrailed-2 mutant mice suggest two separate patterning events during cerebellar development. J Neurosci 17:7881-7889.

Leclerc N, Gravel C, Hawkes R (1988) Development of parasagittal zonation in the rat cerebellar cortex: MabQ113 antigenic bands are created postnatally by the suppression of antigen expression in a subset of Purkinje cells. J Comp Neurol 273:399-420.

Leclerc N, Schwarting GA, Herrup K, Hawkes R, Yamamoto M (1992) Compartmentation in mammalian cerebellum: zebrin II and P-path antibodies define three classes of sagittally organized bands of Purkinje cells. Proc Natl Acad Sci USA 89:5006-5010.

Millen KJ, Wurst W, Herrup K, Joyner AL (1994) Abnormal embryonic cerebellar development and patterning of postnatal foliation in two mouse Engrailed-2 mutants. Development 120:695-706.

Millen KJ, Chi-Chung H, Joyner AL (1995) A role for En-2 and other murine homologs of Drosophila segment polarity genes in regulating positional information in the developing cerebellum. Development 121:3935-3945.

Oberdick J, Schilling K, Smeyne RJ, Corbin JG, Bocchiaro C, Morgan JI (1993) Control of segment-like patterns of gene expression in the mouse cerebellum. Neuron 10:1007-1018.

Oberdick J, Baader SL, Schilling K (1998) From zebra stripes to postal 
zones: deciphering patterns of gene expression in the cerebellum. Trends Neurosci 21:383-390.

Oster-Granite ML, Herndon RM (1976) The pathogenesis of parvovirus-induced cerebellar hypoplasia in the syrian hamster, $M e$ socricetus auratus. Fluorescent antibody, foliation, cytoarchitectonic, Golgi and electron microscopic studies. J Comp Neurol 169:481-521.

Paradies MA, Grishkat H, Smeyne RJ, Oberdick J, Morgan JI, Eisenman LM (1996) Correspondence between L7-lacZ-expressing Purkinje cells and labeled olivocerebellar fibers during late embryogenesis in the mouse. J Comp Neurol 374:451-466.

Penn AA, Riquelme PA, Feller MB, Shatz CJ (1998) Competition in retinogeniculate patterning driven by spontaneous activity. Science 279:2108-2112.

Rétaux S, McNeill L, Harris WA (1996) Engrailed, retinotectal targeting, and axonal patterning in the midbrain during Xenopus development: an antisense study. Neuron 16:63-75.

Sanlioglu S, Zhang X, Baader SL, Oberdick J (1998) Regulation of a Purkinje cell-specific promoter by homeodomain proteins: repression by Engrailed-2 vs. synergistic activation by HoxA5 and HoxB7. J Neurobiol 36:559-571.

Schilling K, Schmidt HHHW, Baader SL (1994) Nitric oxide synthase expression reveals compartments of cerebellar granule cells and suggests a role for mossy fibers in their development. Neuroscience 59:893-903.

Seil FJ, Johnson ML, Hawkes R (1995) Molecular compartmentation expressed in cerebellar cultures in the absence of neuronal activity and neuron-glia interactions. J Comp Neurol 356:398-407.

Siegler MVS, Jia XX (1999) Engrailed negatively regulates the expres- sion of cell adhesion molecules connectin and neuroglian in embryonic Drosophila nervous system. Neuron 22:265-276.

Smeyne RJ, Oberdick J, Schilling K, Berrebi AS, Mugnaini E, Morgan JI (1991) Dynamic organization of developing Purkinje cells revealed by transgene expression. Science 254:719-721.

Smeyne RJ, Chu T, Lewin A, Bian F, Crisman SS, Kunsch C, Lira SA, Oberdick J (1995) Local control of granule cell generation by cerebellar Purkinje cells. Mol Cell Neurosci 6:230-251.

Vogel MW, Prittie J (1994) Topographic spinocerebellar mossy fiber projections are maintained in the lurcher mutant. J Comp Neurol 343:341-351.

Vogel MW, Ji Z, Millen K, Joyner AL (1996) The Engrailed-2 homeobox gene and patterning of spinocerebellar mossy fiber afferents. Dev Brain Res 96:210-218.

Voogd J, Bigaré F (1980) Topographical distribution of olivary and cortico nuclear fibers in the cerebellum: a review. In: The inferior olivary nucleus: anatomy and physiology (Courville J, ed), pp 207-235. New York: Raven.

Wassef M, Zanetta JP, Brehier A, Sotelo C (1985) Transient biochemical compartmentalization of Purkinje cells during early cerebellar development. Dev Biol 111:129-137.

Wassef M, Sotelo C, Thomasset M, Granholm A, Leclerc N, Rafrafi J, Hawkes R (1990) Expression of compartmentation antigen zebrin I in cerebellar transplants. J Comp Neurol 294:223-234.

Wurst W, Auerbach AB, Joyner AL (1994) Multiple developmental defects in Engrailed-1 mutant mice: an early mid-hindbrain deletion and patterning defects in forelimbs and sternum. Development 120: 2065-2075. 